\title{
THREE-DIMENSIONAL DOUBLE-DIFFUSIVE NATURAL CONVECTION WITH OPPOSING BUOYANCY EFFECTS IN POROUS ENCLOSURE BY BOUNDARY ELEMENT METHOD
}

\author{
J. KRAMER, J. RAVNIK, R. JECL \& L. ŠKERGET \\ Faculty of Civil Engineering, Faculty of Mechanical Engineering, University of Maribor, Maribor, Slovenia.
}

\begin{abstract}
A three-dimensional double-diffusive natural convection with opposing buoyancy effects in a cubic enclosure filled with fluid saturated porous media is studied numerically using the boundary element method (BEM). The mathematical model is based on the space-averaged Navier-Stokes equations, which are coupled with the energy and species equations. The simulation of coupled laminar viscous flow, heat and solute transfer is performed using a combination of single-domain BEM and subdomain BEM, which solves the velocity-vorticity formulation of governing equations. The numerical simulations for a case of negative values of buoyancy coefficient are presented, focusing on the situations where the flow field becomes three-dimensional. The results are analyzed in terms of the average heat and mass transfer at the walls of the enclosure. When possible, the results are compared with previous existing numerical data published in literature.
\end{abstract}

Keywords: boundary element method, Brinkman-extended Darcy formulation, porous media, velocity-vorticity formulation, three-dimensional double-diffusive natural convection.

\section{INTRODUCTION}

The analysis of convective flows in porous media has been the subject of intense research over the last few decades. Several published experimental, analytical, as well as numerical results show the importance of the problem, which has several applications in natural and industrial processes. In the field of buoyancy-induced flows, the most commonly studied problems are thermally driven flows, which can simulate, e.g. the heat transport in fibrous insulations, geothermal energy. More challenging situations occur in the case of combined action of thermal and concentration buoyancy forces, which can aid or oppose each other, in general. The so-called double-diffusive natural convection occurs in various engineering processes, e.g. contaminant transport in groundwater, heat and mass transfer in the mushy zone arising during the solidification of alloys. In such processes, complex flow patterns may form mainly due to the presence of porous media, which adds hydraulic resistance, as well as the competition between the thermal and concentration buoyancy forces.

The flow in porous enclosures under these circumstances has been investigated mainly for two-dimensional (2D) geometries. However, for a certain range of controlling parameters in an enclosure imposed to thermal and concentration gradient, the flow may become three dimensional (3D).

Several different configurations have been studied considering the double-diffusive natural convection in porous enclosures, which differ from each other regarding the position of the thermal and concentration gradients. Most commonly studied configurations that can be found in the literature include the following $[1,2]$ :

- Thermal and concentration gradients are imposed on vertical walls and are either aiding or opposing each other. 
- Thermal and concentration gradients are imposed on horizontal walls and are either aiding or opposing each other.

- Thermal/concentration gradient is imposed on vertical wall and concentration/thermal gradient is imposed on the horizontal wall.

Most published studies dealing with double-diffusive natural convection in porous media are based on the 2D geometry and mainly on situations where thermal and concentration buoyancy forces are aiding each other [3-10]. Combined natural convection with opposing buoyancy effects are reported in [11,12]. Only few recent studies are considering 3D geometry. Sezai and Mohamad [13] published study where 3D double-diffusive natural convection in porous media is considered, where the thermal and concentration buoyancy forces are opposing each other. They reported that under a certain range of controlling parameters (porous Rayleigh number, Lewis number, buoyancy coefficient), the flow in cubic enclosure becomes three dimensional. Later Mohamad et al. [14] investigated 3D convection flows in an enclosure subjected to opposing thermal and concentration gradients, focusing on the influence of the lateral aspect ratio. They found that for the certain range of controlling parameters, the aspect ratio has no influence on the rates of heat and mass transfer, but it strongly influences the flow structure.

In the present paper, a combination of single domain and subdomain boundary element method is presented for simulation of double-diffusive natural convection in a cubic enclosure. The algorithm solves the velocity-vorticity formulation of the space averaged Navier-Stokes equations, which are obtained for porous media flow. The main advantage of the proposed numerical scheme, as compared with classical volume-based numerical methods, is that it offers an effective way of dealing with boundary conditions on the solid walls when solving the vorticity equation. Namely, the boundary vorticity is computed directly from the kinematic part by a single-domain boundary element method (BEM) and not through the use of some approximate formulae.

The proposed algorithm is based on the pure fluid and nanofluid simulation codes obtained by Ravnik et al. $[15,16]$. Numerical examples for different values of buoyancy ratio at fixed porous Rayleigh, Darcy, and Lewis numbers are investigated, focusing on situations where thermal and concentration buoyancy forces are opposing each other (negative values of buoyancy coefficient).

\section{MATHEMATICAL FORMULATION}

The geometry under consideration is a cubic enclosure, filled with porous media, which is fully saturated with fluid and is shown in Fig. 1. The left and right vertical walls are imposed to different temperature and concentration values, where $T_{1}>T_{2}$ and $C_{1}>C_{2}$, while the remaining boundaries are adiabatic and impermeable. It is assumed that the fluid is incompressible, Newtonian and the fluid flow is steady and laminar. Furthermore, the porous matrix is assumed to be homogenous, isotropic, and non-deformable. The porosity and permeability of porous medium are constant, while the density depends only on temperature and concentration variations and can be described with Oberbeck Boussinesq approximation.

Due to subjected temperature and concentration differences on two vertical walls, the natural convection phenomena in the enclosure will occur. The density of the heated fluid next to the hot wall decreases and buoyancy will carry it upwards. On the other hand, fluid along the cold wall will be colder and denser, and it will travel downwards. Due to 


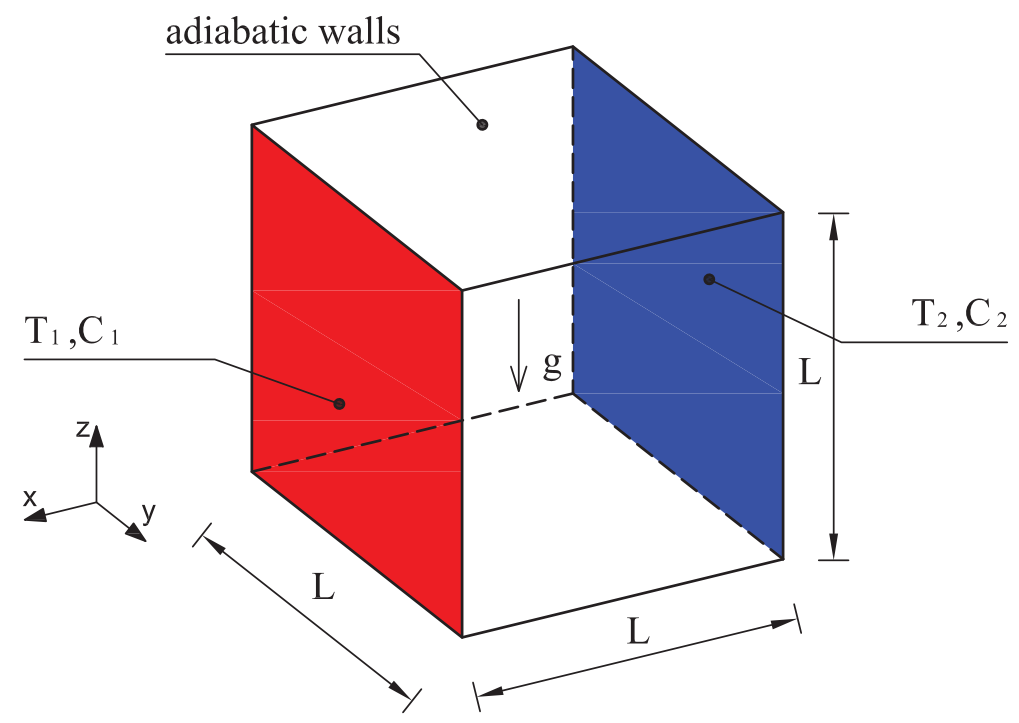

Figure 1: Geometry of the problem.

applied concentration differences on the walls, additional concentration buoyancy forces are induced, which cause additional movement of the fluid. Both induced buoyancy forces can aid or oppose each other, which also influences the strength of the convective motion of the fluid. The cases when solute is transported due to induced temperature gradient (Soret effect) or heat is transferred due to concentration gradient (Dufour effect) have been neglected in the present study.

\subsection{Governing equations}

The governing equations for the problem of double-diffusive natural convection in porous media are given in terms of conservation laws for mass, momentum, energy, and species. They are obtained from classical Navier-Stokes equations for the pure fluid flow, which are generally written at the microscopic level. By volume averaging over suitable representative elementary volume and considering the fact that only a part of this volume, expressed with the porosity $\phi$, is available for fluid flow, macroscopic or volume averaged Navier-Stokes equations can be derived. The averaging procedure is given in detail in [17]. The general set of macroscopic conservation equations can be written as:

- continuity equation

$$
\vec{\nabla} \cdot \vec{v}=0
$$

- momentum equation

$$
\frac{1}{\phi} \frac{\partial \vec{v}}{\partial t}+\frac{1}{\phi^{2}}(\vec{v} \cdot \vec{\nabla}) \vec{v}=-\left(\beta_{T}\left(T-T_{0}\right)+\beta_{C}\left(C-C_{0}\right) \vec{g}-\frac{1}{\rho_{0}} \vec{\nabla} p+\frac{1}{\phi} v \nabla^{2} \vec{v}-\frac{v}{K} \vec{v},\right.
$$


- energy equation

$$
\sigma \frac{\partial T}{\partial t}+(\vec{v} \cdot \vec{\nabla}) T=\lambda_{e} / c_{f} \nabla^{2} T
$$

- species equation

$$
\phi \frac{\partial C}{\partial t}+(\vec{v} \cdot \vec{\nabla}) C=D \nabla^{2} C
$$

The parameters used above are: $\vec{v}$ volume averaged velocity, $\phi$ porosity, $t$ time, $\rho$ density, $v$ kinematic viscosity, $p$ pressure, $\vec{g}$ gravity vector, and $K$ permeability. In the energy equation, $\sigma$ represents the heat capacity ratio $\sigma=\left(\phi c_{f}+(1-\phi) c_{s}\right) / c_{f}$, where $c_{f}=\left(\rho c_{p}\right)_{f}$ and $c_{s}=\left(\rho c_{p}\right)_{s}$ are heat capacities for fluid and solid phases, respectively. $\lambda_{e}$ is the effective thermal conductivity of the fluid saturated porous media given as $\lambda_{e}=\phi \lambda_{f}+(1-\phi) \lambda_{s}$, where $\lambda_{f}$ and $\lambda_{s}$ are thermal conductivities for fluid and solid phases, respectively. In the species equation $C$ is concentration, and $D$ is mass diffusivity. The momentum equation is coupled with energy and species equation due to the buoyancy term, which is described with the Oberbeck Boussinesq approximation, considering the fact that the fluid density depends only on temperature and concentration variations:

$$
\rho=\rho_{0}\left(1-\beta_{T}\left(T-T_{0}\right)-\beta_{C}\left(C-C_{0}\right)\right)
$$

$\beta_{T}$ and $\beta_{C}$ in the above equation are volumetric thermal expansion coefficient and volumetric expansion coefficient due to chemical species, respectively:

$$
\beta_{T}=-\frac{1}{\rho}\left[\frac{\partial \rho}{\partial T}\right]_{C}, \quad \beta_{C}=-\frac{1}{\rho}\left[\frac{\partial \rho}{\partial C}\right]_{T}
$$

The subscript 0 refers to a reference state.

In the present work, the velocity-vorticity formulation of macroscopic Navier-Stokes equations is used, obtained with the introduction of the vorticity vector as a curl of the velocity field $\vec{\omega}=\vec{\nabla} \times \vec{v}$, which is solenoidal by the definition $\vec{\nabla} \cdot \vec{\omega}=0$. Due to velocity-vorticity formulation, the computational scheme is partitioned into kinematic and kinetic parts, where the kinematics is governed by the elliptic velocity vector equation, while the kinetics consists of vorticity transport equation [18].

Non-dimensional form of the governing equations is adopted, using following dimensionless variables:

$$
\begin{aligned}
& \vec{v} \rightarrow \frac{\vec{v}}{v_{0}}, \vec{r} \rightarrow \frac{\vec{r}}{L}, \vec{\omega} \rightarrow \frac{\vec{\omega} L}{v_{0}}, t \rightarrow \frac{v_{0} t}{L}, t_{\omega} \rightarrow \frac{v_{0} t_{\omega}}{L}, t_{T} \rightarrow \frac{v_{0} t_{T}}{L}, \\
& t_{C} \rightarrow \frac{v_{0} t_{C}}{L}, T \rightarrow \frac{T-T_{0}}{\Delta T}, C \rightarrow \frac{C-C_{0}}{\Delta C}, \vec{g} \rightarrow \frac{\vec{g}}{g_{0}},
\end{aligned}
$$

where $v_{0}$ is characteristic velocity, $\vec{r}$ position vector, $L$ characteristic length, $\vec{\omega}$ vorticity vector, $t$ time. $t_{\omega}, t_{T}$, and $t_{C}$ are modified times as $t_{\omega}=t / \phi, t_{T}=t / \sigma$, and $t_{T}=t / \phi$. Furthermore, $T_{0}$ and $C_{0}$ are characteristic temperature and concentration, $\Delta T$ and $\Delta C$ are characteristic temperature and concentration differences. Characteristic velocity is given with the 
expression $v_{0}=\lambda_{f} /\left(c_{f} L\right)$, which is common definition when considering buoyant flow simulations.

The kinematics equation, which is a vector elliptic partial differential equation of Poisson type can be written for the case of an incompressible fluid as:

$$
\nabla^{2} \vec{v}+\vec{\nabla} \times \vec{\omega}=0
$$

where both velocity and vorticity are divergence free.

The kinetics is governed by the macroscopic vorticity transport equation, energy, and species equations.

The macroscopic non-dimensional vorticity equation can be written as:

$$
\frac{\partial \vec{\omega}}{\partial t_{\omega}}+(\vec{v} \cdot \vec{\nabla}) \vec{\omega}=(\vec{\omega} \cdot \vec{\nabla}) \vec{v}-\operatorname{PrR}_{T} \phi^{2} \vec{\nabla} \times(T+N C) \vec{g}+\operatorname{Pr} \phi \nabla^{2} \vec{\omega}-\frac{\operatorname{Pr}}{D a} \phi^{2} \vec{\omega},
$$

with non-dimensional governing parameters defined as:

- $P r$, Prandtl number

$$
\operatorname{Pr}=\frac{v}{\alpha},
$$

Where $v$ is kinematic viscosity and $\alpha$ thermal diffusivity, given as $\alpha=\lambda f / c_{f}$.

- $R a_{T}$ thermal fluid Rayleigh number:

$$
R a_{T}=\frac{g \beta_{T} \Delta T L^{3}}{v \alpha}
$$

- $N$, buoyancy coefficient:

$$
N=\frac{R a_{S}}{R a_{T}}
$$

where $R a_{S}$ is solutal Rayleigh number:

$$
R a_{S}=\frac{g \beta_{C} \Delta C L^{3}}{v \alpha}
$$

- $D a$, Darcy number:

$$
D a=\frac{K}{L^{2}},
$$

where $K$ is permeability of porous media.

Furthermore, the porous Rayleigh number is defined as:

$$
R a_{p}=R a_{T} D a=\frac{g_{0} \beta_{T} \Delta T L K}{\nu \alpha} .
$$


Equation (9) is derived from the governing momentum equation applying the curl operator. The advective vorticity transport is equated on the left hand side, while the first and the second terms on the right hand side are vortex twisting and stretching term and the buoyancy term. In this case, the Darcy-Brinkman formulation is used with two viscous terms, e.g. Brinkman viscous term (third on the r.h.s) and Darcy viscous term (fourth on the r.h.s.). The Brinkman viscous term is analogous to the Laplacian term (diffusion term) in the classical Navier-Stokes equations for pure fluid flow. It expresses the viscous resistance or viscous drag force exerted by the solid phase on the flowing fluid at their contact surfaces. With the Brinkman term the non-slip boundary condition on a surface, which bounds porous media is satisfied [1]. The influence of Brinkman viscous term depends on the Darcy number. In case of high $D a$, the Brinkman viscous term plays a significant role and reduces the overall heat transfer. With decrease in $D a$ the influence of Brinkman term becomes almost negligible; consequently, the inertial effect becomes significant due to high fluid velocity. The influence of Darcy term is investigated in [19] for the case of 2D double-diffusive natural convection in porous media and in [20] for the case of 3D natural convection in porous cube.

Further assumption is that no internal energy sources are present in the fluid-saturated porous media. The irreversible viscous dissipation is also neglected, while no highvelocity flow of highly viscous fluid is considered in the present study. The solid phase of porous medium is assumed to be in thermal equilibrium with the saturation fluid. According to this, the energy conservation equation in the non-dimensional form can be written as:

$$
\frac{\partial T}{\partial t_{T}}+(\vec{v} \cdot \vec{\nabla}) T=\frac{\lambda_{e}}{\lambda_{f}} \nabla^{2} T .
$$

The species conservation equation in its non-dimensional form reads:

$$
\frac{\partial C}{\partial t_{C}}+(\vec{v} \cdot \vec{\nabla}) C=L e \nabla^{2} C,
$$

Where $L_{e}$ is Lewis number, given with the expression:

$$
L e=\frac{\alpha}{D} .
$$

\section{BOUNDARY ELEMENT METHOD}

The governing set of equations (8), (9), (16), and (17) are solved using an algorithm based on the BEM. Either Dirichlet or Neumann type boundary conditions for velocity, temperature, and concentration must be known. The no-slip boundary condition is prescribed on all solid walls; in addition, temperature and concentration differences are prescribed on vertical walls. The following steps are performed:

1. The porous media parameters (porosity $\phi$, specific heat $\sigma$, and permeability $K$ ) are determined.

2. Vorticity values on the boundary are calculated by single-domain BEM from the kinematics equation (8). 
3. Velocity values within the domain are calculated by subdomain BEM from the kinematics equation (8).

4. Temperature values within the domain are calculated by subdomain BEM form equation (16).

5. Concentration values within the domain are calculated by subdomain BEM from the concentration equation (17).

6. Vorticity values within the domain are calculated by subdomain BEM from the vorticity equation (9).

7. Check convergence. All steps from 2 until 5 are repeated until all flow fields achieve the required accuracy.

The numerical algorithm for simulation of 3D fluid flow by a combination of single and subdomain BEM was developed by Ravnik et al. [15]. The solver has been adopted for simulation of flow, heat, and solute transfer within the porous media. Porous parameters have been introduced in the vorticity, temperature, and concentration equations.

\subsection{Integral form of governing equations}

All governing equations are written in the integral form by using the Green's second identity for the unknown field function and the fundamental solution $u^{*}$ of the diffusion operator $u^{*}=1 / 4 \pi|\vec{\xi}-\vec{r}|$.

The integral form of the kinematics equation in its tangential form is:

$$
\begin{array}{r}
c(\vec{\xi}) \vec{n}(\vec{\xi}) \times \vec{v}(\vec{\xi})+\vec{n}(\vec{\xi}) \times \int_{\Gamma} \vec{v} \vec{\nabla} u^{*} \cdot \vec{n} d \Gamma \\
=\vec{n}(\vec{\xi}) \times \int_{\Gamma} \vec{v} \times(\vec{n} \times \vec{\nabla}) u^{*} d \Gamma+\vec{n}(\vec{\xi}) \times \int_{\Omega}\left(\vec{\omega} \times \vec{\nabla} u^{*}\right) d \Omega .
\end{array}
$$

Here, $\vec{\xi}$ is the source or collocation point, $\vec{n}$ is a vector normal to the boundary, pointing out of the domain, and $c(\vec{\xi})$ is the geometric factor defined as $c(\vec{\xi})=\theta / 4 \pi$, where $\theta$ is the inner angle with origin in $\vec{\xi}$. This tangential form of the kinematics equation is used to determine boundary vorticity values; the domain vorticity and velocity values are taken from the previous nonlinear iteration. In addition, the domain velocity values are obtained from the kinematics equation, where the following form is used:

$$
c(\xi) \vec{v}(\xi)+\int_{\Gamma} \vec{v}(\vec{n} \cdot \vec{\nabla}) u^{*} d \Gamma=\int_{\Gamma} \vec{v} \times(\vec{n} \times \vec{\nabla}) u^{*} d \Gamma+\int_{\Omega}\left(\vec{\omega} \times \vec{\nabla} u^{*}\right) d \Omega .
$$

Solution is obtained by subdomain BEM. Boundary values of velocity are known boundary conditions, while domain values of vorticity are assumed known from the previous iteration.

In addition, the same fundamental solution and a standard BEM derivation [21] are used to write the integral forms of the vorticity transport equation (9), energy transport eqn (16), and species transport eqn (17):

$$
\begin{array}{r}
c(\vec{\xi}) \omega_{j}(\vec{\xi})+\int_{\Gamma} \omega_{j} \vec{\nabla} u^{*} \cdot \vec{n} d \Gamma=\int_{\Gamma} u^{*} q_{j} d \Gamma+ \\
+\frac{1}{\operatorname{Pr}} \frac{1}{\phi}\left(\int_{\Gamma} \vec{n} \cdot\left\{u^{*}\left(\vec{v} \omega_{j}-\vec{\omega} v_{j}\right)\right\} d \Gamma-\int_{\Omega}\left(\vec{v} \omega_{j}-\vec{\omega} v_{j}\right) \cdot \vec{\nabla} u^{*} d \Omega\right)- \\
-R a_{T} \phi \int_{\Gamma}\left(u^{*} T \vec{g} \times \vec{n}\right)_{j} d \Gamma-R a_{T} \phi \int_{\Omega}\left((T+N C) \vec{\nabla} \times u^{*} \vec{g}\right)_{j} d \Omega+\frac{\phi}{D a} \int_{\Omega} \omega_{j} u^{*} d \Omega,
\end{array}
$$




$$
\begin{array}{r}
c(\vec{\xi}) T(\vec{\xi})+\int_{\Gamma} T \vec{\nabla} u^{*} \cdot \vec{n} d \Gamma=\int_{\Gamma} u^{*} q_{T} d \Gamma+ \\
+\frac{\lambda_{f}}{\lambda_{e}}\left(\int_{\Gamma} \vec{n} \cdot\left\{u^{*}(\vec{v} T)\right\} d \Gamma-\int_{\Omega}(\vec{v} T) \cdot \vec{\nabla} u^{*} d \Omega\right) . \\
c(\vec{\xi}) C(\vec{\xi})+\int_{\Gamma} C \vec{\nabla} u^{*} \cdot \vec{n} d \Gamma=\int_{\Gamma} u^{*} q_{C} d \Gamma+ \\
+\frac{1}{L e}\left(\int_{\Gamma} \vec{n} \cdot\left\{u^{*}(\vec{v} C)\right\} d \Gamma-\int_{\Omega}(\vec{v} C) \cdot \vec{\nabla} u^{*} d \Omega\right) .
\end{array}
$$

Here, $\omega_{j}$ is a vorticity component, $q_{j}$ is a component of vorticity flux, $q_{T}$ and $q_{C}$ are the heat and species flux, respectively. In the present study, only steady flow fields will be considered, and thus, the time-derivative terms $\partial \omega / \partial t_{\omega}, \partial T / \partial t_{T}$ and $\partial C / \partial t_{C}$ are omitted.

In the subdomain BEM, a mesh of the entire domain $\Omega$ is used, where each mesh element is named a subdomain. Equations (21), (22), and (23) are written for each of the subdomains. In order to obtain a discrete version of the equations, shape functions are used to interpolate field functions and flux across the boundary inside of the subdomain. In this work, hexahedral subdomains with 27 nodes are used, which enables continuous quadratic interpolation of field functions. The boundary of each hexahedron consists of six boundary elements. On each boundary element, the flux is interpolated using discontinuous linear interpolation scheme with four nodes. By using discontinuous interpolation, flux definition problems in the corners and edges could be avoided. Between subdomains, the functions and their fluxes are assumed to be continuous. The resulting linear systems of equations are over-determined and sparse. They are solved in a least-squares manner.

\section{RESULTS AND DISCUSSION}

Applying temperature and concentration differences on two opposite walls on a cubic cavity, which is otherwise insulated, starts up natural convection phenomena due to aiding or opposing thermal and solutal buoyancy forces, which results in a large vortex in the main part of enclosure. In case when buoyancy coefficient is negative, the buoyancy forces are opposing each other, which slows down the convective motion and at the critical point, the fluid flow direction is reversed. In this paper, the simulations for fixed values of porous Rayleigh, Lewis, and Darcy numbers are presented in order to investigate the influence of negative value of the buoyancy coefficient. All calculations are performed for very low values of Darcy number $\left(D a=10^{-6}\right)$, which means the model gives similar results as the classical Darcy model [22]. Comprehensive studies, patterned on the present research, concerned with the combined heat and solute transfer processes driven by buoyancy through fluid saturated porous medium for 2D geometries using classical Darcy model, were published by Bejan et al. [3--6].

The results are expressed in terms of average Nusselt and Sherwood numbers presenting the wall heat and species flux, which are given as:

$$
N u=\int_{\Gamma} \vec{\nabla} T \cdot \vec{n} d \Gamma, \quad S h=\int_{\Gamma} \vec{\nabla} C \cdot \vec{n} d \Gamma .
$$

All calculations were performed on a nonuniform mesh with $20 \times 8 \times 20$ subdomains and 2,8577 nodes. Subdomains are concentrated toward the hot and cold walls. The convergence criterion for all field functions was $10^{-5}$, and under-relaxation of vorticity, temperature, and concentration values ranging from 0.1 to 0.01 was used. For all calculations, the porosity parameter value was set to $\phi=0.8$, the specific heat $\sigma=1$, and Prantl number 10 . 
Table 1: Nusselt and Sherwood number values for 3D natural convection in a cubic enclosure for $D a=10^{-6}$ and different values of porous Rayleigh number, Lewis number and buoyancy coefficient. The results are compared to study of Mohamad et al. [14] (values in brackets).

\begin{tabular}{lcc}
\hline$R a_{p}=10, N=-0,5$ & $L e=1$ & $L e=10$ \\
\hline$N u$ & $1,019(1,0198)$ & $1,039(1,0404)$ \\
$S h$ & $1,019(1,0198)$ & $2,450(2,4467)$ \\
\hline$L e=50, N=-0,2$ & $R a_{p}=1$ & $R a_{p}=10$ \\
\hline$N u$ & $1,001(1,0005)$ & $1,072(1,0705)$ \\
$S h$ & $1,952(1,9517)$ & $7,388(6,9861)$ \\
\hline
\end{tabular}

Table 2: Nusselt and Sherwood number values for 3D natural convection in a cubic enclosure for $R a_{p}=100$, Le $=10, D a=10^{-6}$ and different values of buoyancy coefficient.

\begin{tabular}{|c|c|c|c|c|c|c|}
\hline & & $R a_{p}=100$ & $D a=10-6$ & $L e=10$ & & \\
\hline$N=1$ & $N=0$ & $N=-0.5$ & $N=-1$ & $N=-1.5$ & $N=-2$ & $N=-3$ \\
\hline$\overline{N u} 4,364$ & 3,4358 & 2,811 & 2,068 & 1.291 & 1,061 & 1,893 \\
\hline Sh 18,444 & 13,295 & 10,233 & 7,653 & 7.449 & 9,122 & 13,923 \\
\hline
\end{tabular}

In order to validate the obtained numerical algorithm, some test examples were performed first and compared with the available results from the literature. Table 1 presents Nusselt and Sherwood number values for $D a=10^{-6} ; R a_{p}=1$ and 10; $L e=1,10$, and 50; and $N=-0,2$ and 0, 5. The comparison with the study of Mohamad et al. [14] shows good agreement.

According to published study of Sezai and Mohamad [13], where 3D analysis of double-diffusive natural convection in a porous enclosure is investigated, focusing on situations, where the temperature and concentration gradients are opposing each other, the flow becomes 3D for a certain range of controlling parameters, e.g. for a fixed $L e=10, R a>20$, and $N=-0.5$. From this starting point, further presented results were obtained for fixed values of $R a_{p}=100, D a=10^{-6}$, and $L e=10$ and variable negative values of $N$. The results of computation are presented in Table 2. It is observed that with the decrease of $N$ from 1 until -2 , the overall heat and mass transfer decrease, which clearly shows the influence of opposite directions of thermal and solutal buoyancy forces. In case when $N=-2$, the Nusselt number value is near to unity, which means that heat is transferred mainly by the conduction mechanism. The values of Sherwood number start to increase from $N=-1.5$, where the solutal buoyancy force is predominant. These results are consistent with the temperature, concentration, and flow fields on plane $y=0.5$, presented in Figs. 2, 3, and 4. Both the temperature and concentration fields are observed to be stratified for higher values of $N$. Decrease of $N$ slows down the convective motion, which results in vertical isotherms (when $N=-2$ ) and iso-concentration lines (when $N=-1.5)$. The flow direction is clearly reversed when $N=-1.5$ due to downward species 

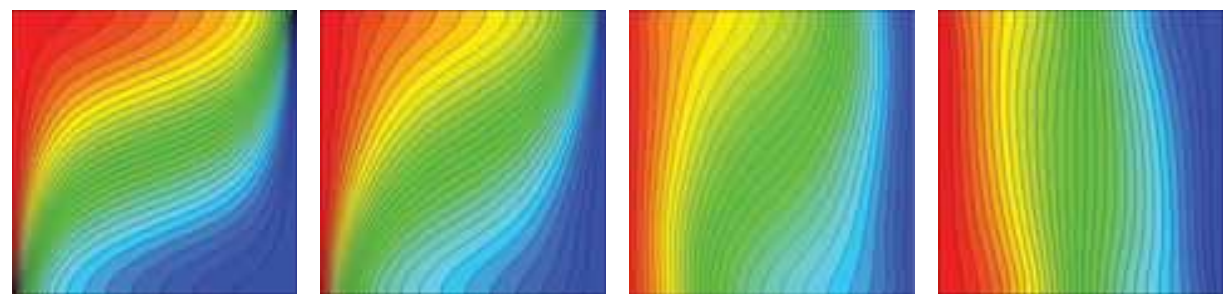

Figure 2: Temperature contour plots on the $y=0.5$ plane for $R a_{P}=100, D a=10^{-6}, L e=10$ and $N=0, N=-1, N=-1.5$ and $N=-2$, respectively.
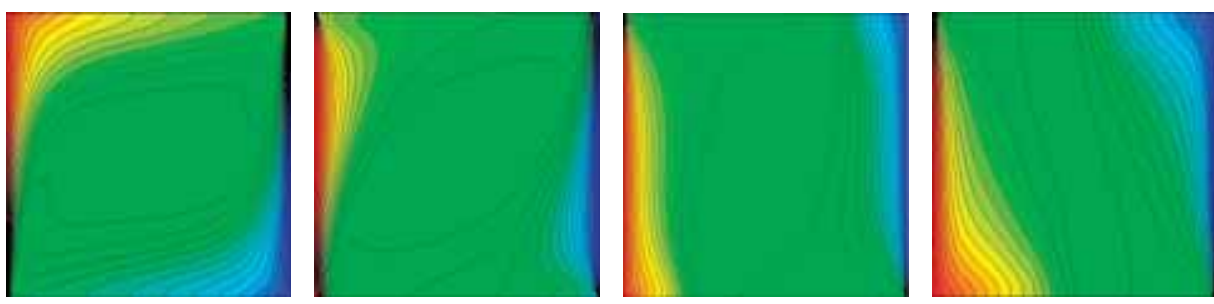

Figure 3: Concentration contour plots on the $y=0.5$ plane for $R a_{P}=100, D a=10^{-6}, L e=10$ and $N=0, N=-1, N=-1.5$ and $N=-2$, respectively.
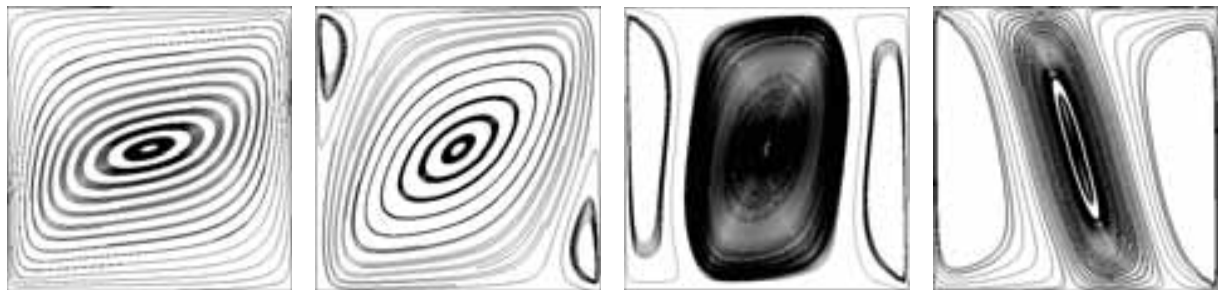

Figure 4: Streamlines on the $y=0.5$ plane for $R a_{P}=100, D a=10^{-6}, L e=10$ and $N=0$, $N=-1, N=-1.5$ and $N=-2$, respectively.

buoyancy, which dominates the flow. This can be clearly seen from the flow field presented in Fig. 4.

In Fig. 5, temperature and concentration profiles for $R a_{P}=100, D a=10^{-6}, L e=10$ and $N=0, N=-0.5, N=-1$, and $N=-2$ are presented. The temperature and concentration gradients increase with the decrease of $N$. The highest gradients can be observed close to the hot and cold walls in case when $N=0$. When $N=-2$, the temperature profile is close to the linear profile. The Nusselt number value is close to unity, which means the heat is transferred mainly by conduction.

The 3D nature of the phenomena can be observed in the corners of the domain, which can be clearly seen from the iso-surfaces of absolute value of $y$ velocity component plotted in Fig. 6. The extent of movement perpendicular to the plane of the main vortex becomes more apparent in case of lower values of $N$. 

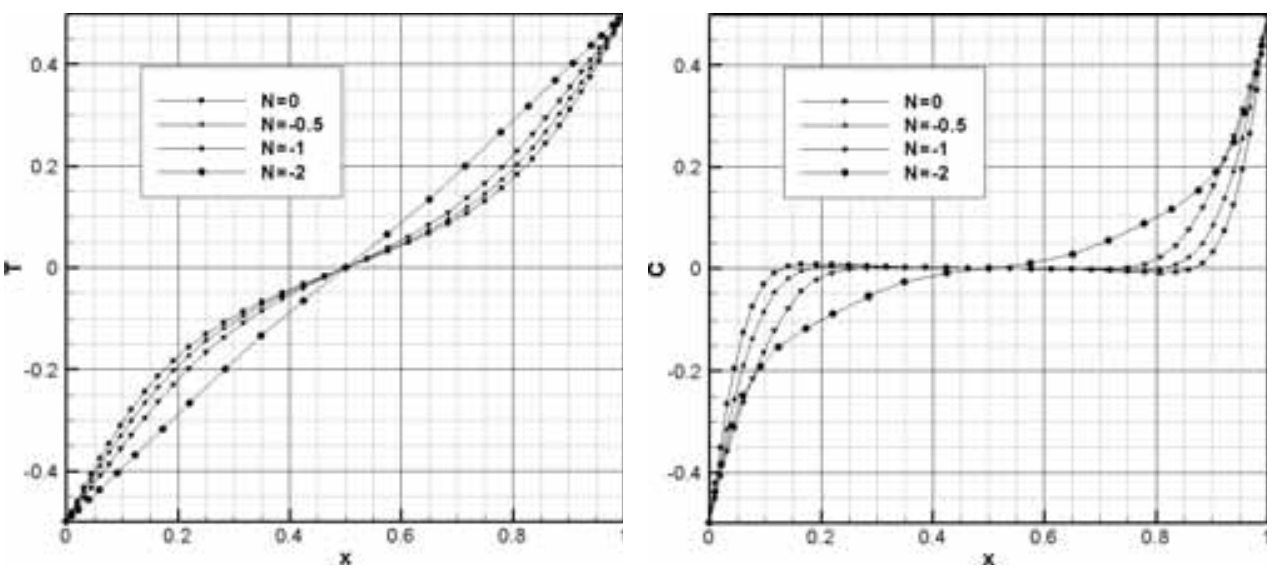

Figure 5: Temperature and concentration profiles at $y=0.5$ and $z=0.5$.
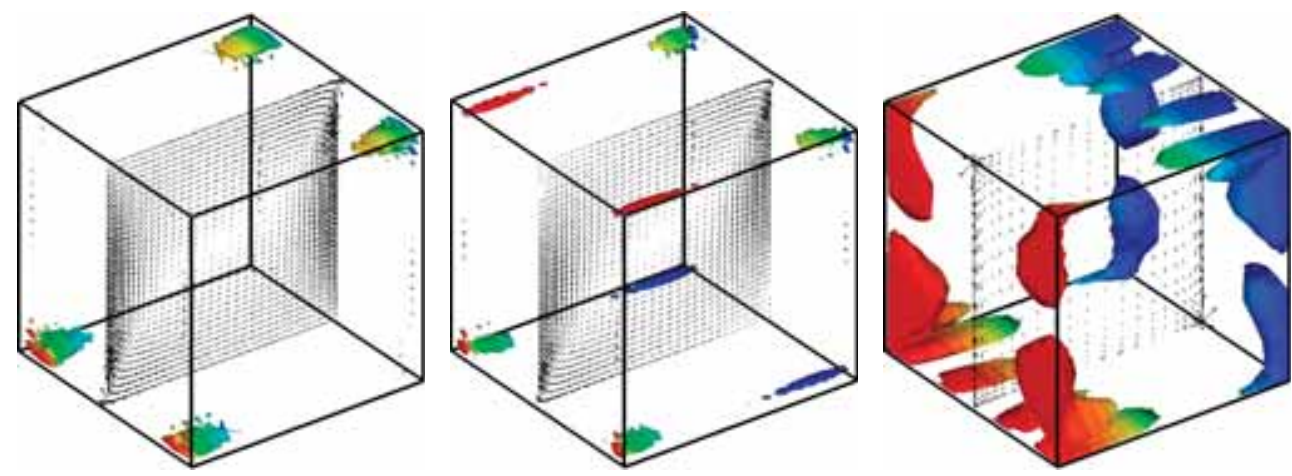

Figure 6: Iso-surfaces with temperature contours $(-0.5<T<0.5)$ for $R a_{p}=100, D a=10^{-6}$, $L e=10$ and absolute value of velocity component $\left|v_{y}\right|=0.5$ for $N=0$ (left), $N=-0.5$ middle and $N=-2$ right. In addition, the velocity vectors on the plane $y=0.5$ are displayed.

\section{SUMMARY}

The 3D natural convection in a cube enclosure filled with fluid saturated porous media was examined numerically using the combination of the single-domain and subdomain boundary element method. The results of overall heat and mass transfer through enclosure are presented, focusing on the influence of opposing effect of thermal and concentration buoyancy forces. The flow as well as heat and mass transfer follow complex patterns depending on the interaction of the governing parameters, especially buoyancy ratio. The local direction of the flow changes because of the opposing buoyant mechanisms, which is also reflecting in the values of Nusselt and Sherwood numbers. Fluid flow becomes threedimensional in case of low values of buoyancy coefficient, which can be observed in the corners of the enclosure. 


\section{ACKNOWLEDGEMENTS}

One of the authors (J. Kramer) acknowledges the financial support to the research project Z2-2035 as provided by the Slovenian Research Agency ARRS.

\section{REFERENCES}

[1] Nield, D.A., \& Bejan, A., Convection in Porous Media, (3rd edn., Springer, 2006.

[2] Vafai, K., Handbook of Porous Media. Taylor \& Francis, 2005. doi: http://dx.doi.org/ $\underline{10.1201 / 9780415876384}$

[3] Bejan, A. \& Khair, K.R., Heat and mass transfer by natural convection in a porous medium. International Journal of Heat and Mass Transfer, 28, pp. 909-918, 1985. doi: http://dx.doi.org/10.1016/0017-9310(85)90272-8

[4] Trevisan, O.V. \& Bejan, A., Natural convection with combined heat and mass transfer buoyancy effects in a porous medium. International Journal of Heat and Mass Transfer, 28, pp. 1597-1611, 1985. doi: http://dx.doi.org/10.1016/0017-9310(85)90261-3

[5] Trevisan, O.V. \& Bejan, A., Mass and heat transfer by natural convection in a vertical slot filled with porous medium. International Journal of Heat and Mass Transfer, 29, pp. 403-415, 1986. doi: http://dx.doi.org/10.1016/0017-9310(86)90210-3

[6] Trevisan, O.V. \& Bejan, A., Combined heat and mass transfer by natural convection in a porous medium. Advances in Heat Transfer, 20, pp. 315-348, 1990. doi: http://dx.doi. org/10.1016/S0065-2717(08)70029-7

[7] Alavyoon, F., On natural convection in vertical porous enclosures due to prescribed fluxes of heat and mass at the vertical boundaries. International Journal of Heat and Mass Transfer, 36, pp. 2479-2498, 1993. doi: http://dx.doi.org/10.1016/S00179310(05)80188-7

[8] Mamou, M., Vasseur, P. \& Bilgen, E., Multiple solutions for double diffusive convection in a vertical porous enclosure. International Journal of Heat and Mass Transfer, 38, pp. 1787-1798, 1995. doi: http://dx.doi.org/10.1016/0017-9310(94)00301-B

[9] Goyeau, B., Songbe, J.-P. \& Gobin, D., Numerical study of double-diffusive natural convection in a porous cavity using the darcy-brinkman formulation. International Journal of Heat and Mass Transfer, 39, pp. 1363-1378, 1996. doi: http://dx.doi.org/ 10.1016/0017-9310(95)00225-1

[10] Nithiarasu, P., Seetharamu, K. N. \& Sundararajan, T., Double-diffusive natural convection in an enclosure filled with fluid-saturated porous medium: a generalized non-darcy approach. Numerical Heat Transfer, 30, pp. 413-426, 1996. doi: http://dx. doi.org/10.1080/10407789608913848

[11] Alavyoon, F., Masuda, Y. \& Kimura, S., On natural convection in vertical porous enclosures due to opposing fluxes of heat and mass prescribed at the vertical walls. International Journal of Heat and Mass Transfer 37, pp. 195-206, 1994. doi: http:// dx.doi.org/10.1016/0017-9310(94)90092-2

[12] Angirasa, D., Peterson, G.P. \& Pop, I., Combined heat and mass transfer by natural convection with opposing buoyancy effects in a fluid saturated porous medium. International Journal of Heat and Mass Transfer, 40, pp. 2755-2773, 1997. doi: http:// dx.doi.org/10.1016/S0017-9310(96)00354-7

[13] Sezai, I. \& Mohamad, A.A., Double diffusive convection in a cubic enclosure with opposing temperature and concentration gradient. Physics of Fluids, 12, pp. 2210-2223, 2000. doi: http://dx.doi.org/10.1063/1.1286422 
[14] Mohamad, A.A., Bennacer, R. \& Azaiez, J., Double diffusion natural convection in a rectangular enclosure filled with binary fluid saturated porous media the effect of lateral aspect ratio. Physics of Fluids, 16, pp. 184-199, 2004. doi: http://dx.doi.org/ $\underline{10.1063 / 1.1630798}$

[15] Ravnik, J., Škerget, L. \& Žunič, Z., Velocity-vorticity formulation for 3D natural convection in an inclined enclosure by BEM. International Journal of Heat and Mass Transfer, 51, pp. 4517-4527, 2008. doi: http://dx.doi.org/10.1016/j.ijheatmasstransfer. 2008.01.018

[16] Ravnik, J., Škerget, L. \& Hriberšek, M., Analysis of three-dimensional natural convection of nanofluids by BEM. Engineering Analysis With Boundary Elements, 34, pp. 1018-1030, 2010. doi: http://dx.doi.org/10.1016/j.enganabound.2010.06.019

[17] Bear, J., Dynamics of Fluids in Porous Media. Dover Publications, Inc.: New York, 1972.

[18] Škerget, L., Hriberšek, M. \& Kuhn, G., Computational fluid dynamics by boundarydomain integral method. International Journal for Numerical Methods in Engineering, 46, pp. 1291-1311, 1999. doi: http://dx.doi.org/10.1002/(SICI)1097-0207(19991120) 46:8<1291::AID-NME755>3.0.CO;2-O

[19] Kramer, J., Jecl, R. \& Škerget, L., Boundary domain integral method for the study of double diffusive natural convection in porous media. Engineering Analysis with Boundary Elements, 31, pp. 897-905, 2007. doi: http://dx.doi.org/10.1016/ j.enganabound.2007.04.001

[20] Kramer, J., Jecl, R., Ravnik, J. \& Škerget, L., Simulation of 3d flow in porous media by boundary element method. Engineering Analysis with Boundary Elements, 35, pp. 1256-1264, 2011. doi: http://dx.doi.org/10.1016/j.enganabound.2011.06.002

[21] Wrobel, L. C., The Boundary Element Method. John Willey \& Sons, LTD, 2002.

[22] Jecl, R., Škerget, L. \& Petrešin, E., Boundary domain integral method for transport phenomena in porous media. International Journal for Numerical Methods in Fluids, 35,pp.39-54,2001.doi:http://dx.doi.org/10.1002/1097-0363(20010115)35:1<39::AIDFLD81>3.0.CO;2-3 